\title{
L'architecture du projet de recherche BIMBY
}

The Architecture of the BIMBY Research Project

\section{David Miet}

\section{(Q) OpenEdition Journals}

Édition électronique

URL : http://journals.openedition.org/crau/585

DOI : $10.4000 /$ crau. 585

ISSN : 2547-5746

\section{Éditeur}

Éditions du patrimoine

\section{Édition imprimée}

Date de publication : 1 novembre 2012

Pagination : 219-224

ISBN : 978-2-7577-0108-9

ISSN : $1296-4077$

\section{Référence électronique}

David Miet, "L'architecture du projet de recherche BIMBY », Les Cahiers de la recherche architecturale et urbaine [En ligne], 26/27 | 2012, mis en ligne le 01 novembre 2017, consulté le 20 avril 2019. URL http://journals.openedition.org/crau/585; DOI : 10.4000/crau.585 
Cet article explicite la méthodologie architecturale employée pour concevoir et mettre en œuvre le projet de recherche BIMBY (« Build In My Back Yard »), dont l'objectif est de permettre l'émergence de stratégies nouvelles d'intensification des tissus pavillonnaires existants.

\section{L'architecture du projet de
recherche BIMBY}

DAVID MIET

\section{Produire des connaissances}

\section{pour évaluer le champ des possibles}

"Et si l'aspiration des Français à la multitude des modes de vie qu'offre

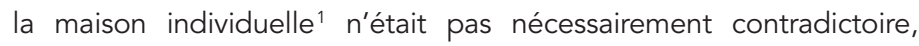
finalement, avec la notion de "ville durable »? Et si les stratégies foncières, immobilières, architecturales, sociales et familiales des habitants s'avéraient potentiellement plus puissantes, en matière de cohésion sociale et de réduction de l'étalement urbain, que les politiques urbaines portées par leurs institutions et mises en œuvre par les aménageurs? Et si les formes bâties les plus décriées par les professionnels de l'urbanisme et de l'architecture, que sont les tissus pavillonnaires des dernières décennies, constituaient en réalité le candidat le plus sérieux ${ }^{2}$, le plus riche et le plus vaste ${ }^{3}$ pour former le terrain sur lequel bâtir les quartiers durables à venir?"

Telles sont les hypothèses " architecturales ${ }^{4}$ que Benoit Le Foll et moi-même avons proposées, fin 2008, à une dizaine de partenaires publics ${ }^{5}$ en vue de constituer une réponse à l'appel à projets « Villes Durables » de

1. Enquêtes après enquêtes, il semble manifeste que la majorité des Français désire vivre en maison individuelle, sans préjuger des raisons qui les emmènent à exprimer aujourd'hui cette préférence.

2. Le choix de terrains en dernière couronne urbaine, la reconquête de terrains pollués ou encore enclavés par le tracé de grandes infrastructures, nous semblent des choix d'implantation d'éco-quartiers peut-être intéressants à certains égards mais finalement anecdotiques aux regards des besoins de construction de logements en France.

3. D'après l'Ordre des architectes, $97 \%$ du foncier alloué chaque année à la construction de logements est destiné à la production d'habitat individuel.

4. Ces grandes hypothèses sont architecturales dans la mesure où 
l'Agence nationale de la recherche. À la suite de nos travaux d'étudiants, puis de plusieurs tentatives infructueuses de lancer une étude sur la « densification pavillonnaire » au sein des groupes de travail du ministère de l'Écologie, cet appel à projets de l'ANR nous est apparu comme l'un des derniers tremplins accessibles pour poursuivre l'exploration des quelques pistes de travail, prometteuses, que nous avaient laissé entrevoir nos premiers travaux sur le sujet.

1/ Considérer l'étalement pavillonnaire d'hier comme un investissement déjà réalisé : les tissus pavillonnaires occupent de vastes territoires déjà viabilisés; ces lieux ne sont-ils pas aujourd'hui stratégiques pour opérer une densification efficace du point de vue économique?

2/ Voir la pression foncière grandissante comme une opportunité : amplifiée par les politiques de lutte contre l'étalement urbain, ne fait-elle pas des propriétaires actuels de maisons individuelles des producteurs de foncier à bâtir en puissance ${ }^{6}$, dans des secteurs souvent déjà desservis par les transports en commun?

3/ Redonner un rôle fort de maître d'ouvrage à I'habitant : pourquoi ne permettrait-on pas aux propriétaires de maisons de détacher et de valoriser une partie de leur terrain comme nouveau terrain à bâtir, afin qu'un autre ménage puisse y construire un logement supplémentaire pour ses propres besoins?

Par le détournement de l'acronyme NIMBY (" Not In My Back Yard ", tout sauf dans mon jardin) en BIMBY ("Build In My Back Yard », construire dans mon jardin) nous avons ainsi invité un certain nombre de collègues architectes, urbanistes, juristes, sociologues, économistes et ingénieurs à nous rejoindre dans la conception d'une nouvelle filière de production de la ville : une filière capable d'intervenir au sein des tissus pavillonnaires existants, qui représentent aujourd'hui la grande majorité des surfaces urbanisées en France.

L'objectif central de la proposition que nous avons soumise à l'ANR, qui sous-tend l'organisation et la complémentarité de l'ensemble des travaux proposés, ainsi que le mode de coordination de ses deux architectes pilotes, garants de l'économie générale de la recherche, peut-être résumé de la façon suivante: produire, en trois ans, des éléments de connaissance suffisants pour engendrer l'émergence de stratégies nouvelles et opérationnelles d'intensification des tissus pavillonnaires existants.

Retenu par l'ANR puis labellisé par le pôle de compétitivité Advancity, le projet BIMBY a ainsi entamé, fin 2009, un programme de travail de trois ans pour un investissement global de 3,1 millions d'euros. Nous décrirons succinctement dans cet article quatre principes méthodologiques que nous nous sommes employés à concevoir, à partager avec nos partenaires et à expérimenter en situation de recherche. Les premiers éléments de résultat du projet nous permettent de penser que cet objectif est d'ores et déjà atteint, à quelques mois de la clôture des travaux du " projet de recherche BIMBY ", et qu'ils permettent même d'envisager aujourd'hui la diffusion d'une « démarche open source BIMBY » qui fait déjà ses premiers pas sur le territoire français.

\section{Analyser un territoire ou évaluer un " gisement "?}

Une multitude d'analyses "objectives " peuvent être réalisées afin de mieux comprendre un territoire en elles ne sont pas « simples» : elles relient les unes aux autres un certain nombre d'hypothèses économiques, sociales, politiques, techniques.

5.Le projet BIMBY rassemble 10 partenaires : y contribuent le Réseau scientifique et technique du ministère du Développement durable, à travers les Centres d'études techniques de l'Équipement, le CETE Normandie-Centre (pilote du projet BIMBY) et le CETE d'Île-de-France (co-pilote), les communautés d'agglomération de Rouen et de Saint-Quentin-en-Yvelines, le Conseil d'architecture, d'urbanisme et de l'environnement de l'Eure et, naturellement, des établissements d'enseignement et leurs laboratoires de recherche : les écoles nationales supérieures d'architecture de Rouen, de Paris-La Villette et de Marseille, le laboratoire Techniques, Territoires et Sociétés (LATTS) et le laboratoire de Recherches Interdisciplinaires Ville, Espace, Société (RIVES). D'autres partenaires supplémentaires sont venus se greffer progressivement à ces travaux, de façon souvent informelle et en adéquation avec la philosophie de diffusion libre des travaux adoptée dès la départ par I'ensemble des partenaires initiaux du projet.

6. Un terrain issu d'une division de terrain se vendra entre 100000 et 300000 euros en île-de-France, entre 30000 et 300000 euros en province. 
fonctionnement : en effet, chaque discipline construit et développe une forme de description ou de modélisation d'un certain plan de la réalité, qui a son propre intérêt. Ces analyses sont en fait si nombreuses, et les degrés d'approfondissement auxquels ont peut les mener si divers, que sans but affiché, aucune d'elles n'est plus pertinente qu'une autre à priori. Ainsi, ces analyses, études préalables et autres éléments de diagnostics sont-ils souvent entrepris de façon mécanique voire automatique selon que l'on réalise un Plan local d'urbanisme, une Zone d'aménagement concertée, une étude de définition ... Pourtant, les moyens extrêmement limités des études urbaines en France $n$ 'invitent-ils pas à choisir avec parcimonie et précaution le type d'analyse de l'existant qu'il convient de conduire dans chaque situation?

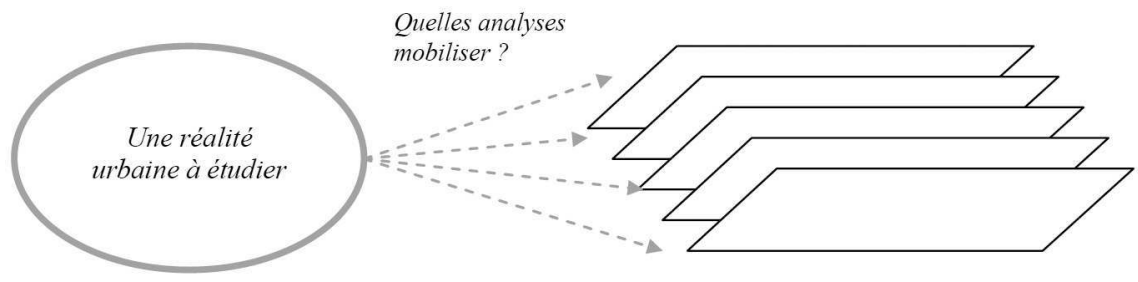

Nous introduisons, dans le projet BIMBY, la notion d'évaluation d'un gisement pour désigner l'analyse ou plutôt l'" éclairage " d'une situation urbaine sous l'angle d'un certain scénario de transformation, lui-même en cours de conception : les spécificités de ce scénario ou de ce modèle d'intervention nécessitent qu'un ensemble d'informations particulières soient réunies afin d'évaluer le potentiel d'un site à évoluer selon ce mode, tandis qu'en retour, ces informations réunies nous aident à mieux concevoir les spécificités du scénario en question.

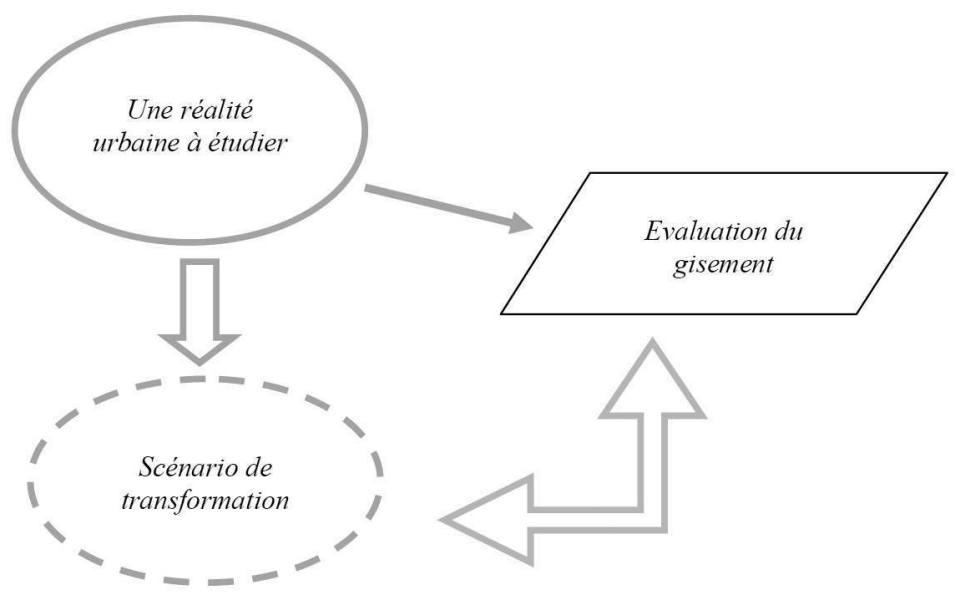


Dans cette perspective, le but de l'analyse n'est plus de décrire la réalité avec une sorte d'objectivité, mais de mettre en lumière un certain nombre de facettes de cette réalité par la description progressive des différents scénarios selon lesquels la situation actuelle pourrait être amenée à évoluer.

Ainsi en va-t-il, par exemple, lorsque l'on se demande quels sont les acteurs capables d'ajouter un nouveau logement sur le terrain d'une maison existante : constructeurs de maisons en diffus, aménageurs et promoteurs n'ont pas les mêmes modèles économiques et, de fait, ne sont pas susceptibles d'intervenir dans les mêmes situations. Dire que dans une partie du territoire, les promoteurs prendraient trop de risque à intervenir, et qu'ils doivent donc laisser la place aux aménageurs ou aux constructeurs en diffus, renseigne sur l'état du marché immobilier local, sur les droits à bâtir qui y sont consentis par le pLu dans la zone concernée, ainsi que sur la perception qu'ont les propriétaires de ces droits à bâtir.

Comme le décrit Thierry Vilmin7, toutes les zones d'une agglomération ne sont pas accessibles à tous les types d'opérateurs. Mais les scénarios - et à fortiori les opérateurs - de la filière BIMBY sont encore à concevoir ${ }^{8}$, si bien que les secteurs d'une agglomération accessibles à cette filière n'ont pas encore de contours bien délimités. Il convient donc d'éclairer petit à petit ce gisement par des scénarios de transformation partiels, que nous pourrons recomposer et décomposer au fur et à mesure de nos travaux.

\section{Décrire des scénarios " partiels » de transformation}

Tout travail interdisciplinaire nécessite un objet de travail en commun. Dans le cadre du projet BIMBY, ces objets ou "modèles" " sont des scénarios partiels de transformation des tissus pavillonnaires existants, notamment des scénarios d'implantation d'une nouvelle maison sur le terrain d'une maison existante. Ce sont ces différents éléments de scénarios qui appelleront le croisement d'analyses sociologiques sur les usages avec des modèles de comportement thermique des maisons par exemple, ou encore la confrontation d'une analyse de morphologie parcellaire avec des modèles qualitatifs d'évaluation des prix immobiliers. Pour un

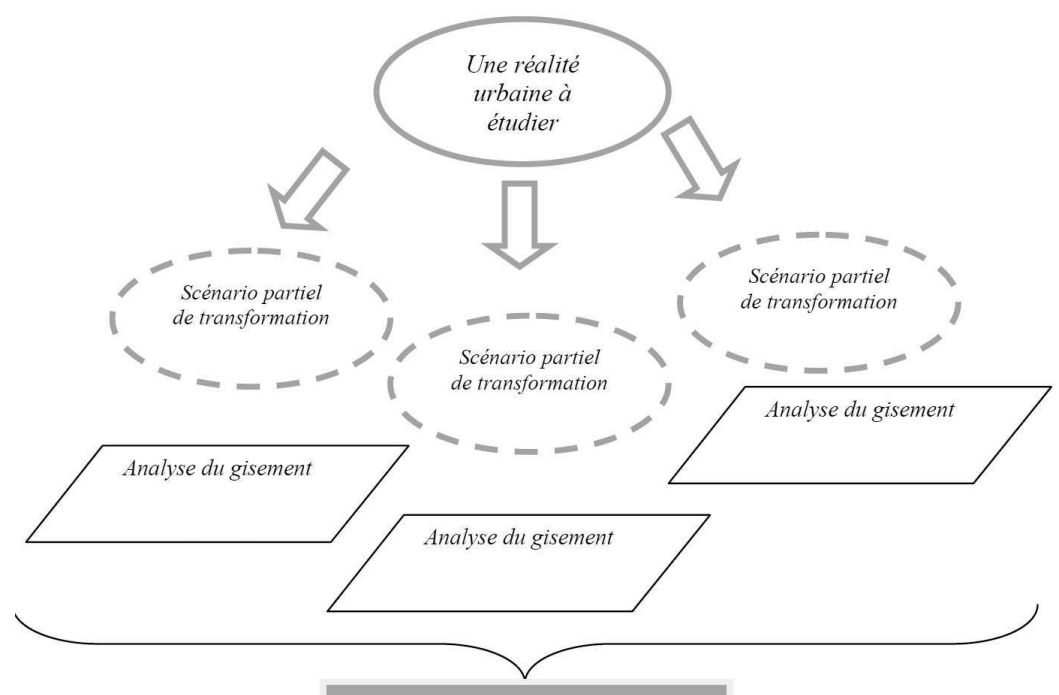

Gisement architectural 
site donné, l'étude de ces scénarios de transformation et les approches croisées qu'ils mobilisent forment ainsi un " gisement architectural » : un éclairage multidimensionnel et modulaire montrant les champs de transformations possibles d'un territoire.

Étudier la possibilité d'ajouter des maisons sur le devant de la rue (scénario partiel $n^{\circ} 1$ ), en créant des parcelles à cheval sur l'espace public et les parcelles privées (scénario partiel $n^{\circ} 2$ ), de telle sorte que la commune récupère une partie de la plus-value foncière réalisée afin de financer des travaux d'organisation du stationnement en entrée de quartier (scénario partiel $n^{\circ} 3$ ), nécessite de connaître, notamment, les espaces publics aujourd'hui surdimensionnés, (élément de gisement $n^{0} 1$ ), les rues dans lesquelles le recul des maisons actuelles par rapport à l'espace public est suffisant (élément de gisement $n^{\circ} 2$ ), celles dans lesquelles la position du système d'assainissement est favorable (élément de gisement $n^{\circ} 3$ ), etc. Cette analyse sera différente et complémentaire de celle évaluant le potentiel du même quartier mais selon un autre scénario de transformation prévoyant, par exemple, l'exploitation de deux bandes de trois mètres de large disponibles de chaque côté d'une limite de voisinage (scénario partiel $n^{\circ} 4$ ) afin de créer une nouvelle parcelle à cheval sur les deux précédentes (scénario partiel $n^{\circ} 5$ ), parcelles sur lesquelles seraient construites des petites maisons à patio (scénario partiel $n^{\circ}$ 6) permettant aux personnes âgées originaires du quartier d'accéder à un logement plus adapté tout en conservant leurs relations de voisinages, leurs habitudes ... (scénario partiel $n^{\circ} 7$ ).
Ainsi, par les éclairages successifs d'une variété de scénarios probables, finit-on par définir, progressivement, le gisement architectural d'un quartier pavillonnaire.

\section{Innover, en décomposant et en recomposant des scénarios partiels}

Formaliser les scénarios de transformation des tissus pavillonnaires existants comme des ensembles de modèles plus simples et assemblables permet d'envisager très rapidement un grand nombre de stratégies innovantes:

- soit en définissant de nouvelles façons de concevoir tel ou tel modèle (une nouvelle façon de rédiger l'article 4 du règlement d'un PLU par exemple) ;

- soit en concevant des associations de scénarios partiels auxquels on n'avait pas encore pensé (combiner telle façon de rédiger l'article 4 d'un PLU avec un modèle de maison porche par exemple, permettant de donner accès à une autre parcelle à l'arrière).

En nous donnant la liberté de faire évoluer les différents modèles les uns par rapport aux autres, le nombre de scénarios possibles augmente considérablement et se pose, naturellement, la question de leur pertinence respective. Pouvons-nous " tester " la validité d'un modèle architectural, c'est-à-dire d'un assemblage de scénarios partiels de transformation? Le parti que nous avons adopté est de mesurer la propension de chaque modèle architectural à être communiqué et intégré à un grand nombre de stratégies plus larges, ce qui est une façon de mesurer sa " concevabilité » ou sa capacité (1) à être pris à nouveau comme un
7. Thierry Vilmin, «Les trois marchés de l'étalement urbain », Études Foncières $n^{\circ}$ 157, mai-juin 2012.

8. À partir, notamment, du modèle de la filière diffuse opérant sur des parcelles issues de division parcellaires.

9. Ces « modèles » peuvent être assimilés aux « pattern » que les architectes Christopher Alexander (en 1977, dans A Pattern Language) puis Kevin Lynch (en 1981, dans Good City Form) ont proposé de prendre comme objets de travail commun. 
scénario partiel de transformation et (2) à être assemblé à d'autres modèles du même genre pour former des scénarios plus complets.

\section{Faire circuler des connaissances assemblables}

La « concevabilité » d'un modèle, c'est-à-dire sa propension à être utilisé dans la pratique pour concevoir des stratégies d'évolution des tissus pavillonnaires, cette concevabilité ne repose pas uniquement sur ses propriétés intrinsèques. Elle dépend, pour une grande partie, de la façon dont le projet de recherche BIMBY envisage d'architecturer et d'outiller la médiation des connaissances produites vers ceux qui pourraient en être les utilisateurs. Pour ce qui concerne les scénarios d'intensification des quartiers pavillonnaires dont nous avons entrepris l'élaboration, un très grand nombre d'acteurs pourraient avoir besoin d'accéder à ces connaissances : les habitants eux-mêmes et leurs familles, notaires et agents immobiliers, architectes et urbanistes, communes et instances supra-communales en charge de la panification territoriale.

Cette question de la médiation des connaissances a été une préoccupation centrale des travaux du projet BIMBY :

- médiation vers les professionnels : la mise en place de la filière BIMBY nécessite l'introduction de nouvelles missions de conception au niveau de plusieurs segments clés de la filière diffuse de production de logements, missions qui seront réalisables dans le cadre d'exercice des métiers actuels ou dans le cadre de nouveaux métiers, qui sont certes encore à inventer mais qui pourront largement s'appuyer sur le répertoire de scénarios partiels d'ores et déjà mis à disposition sur la plateforme open source bimby.fr ;

- médiation vers les particuliers : c'est l'un des verrous méthodologiques qui a été levé à l'occasion des travaux du projet de recherche BIMBY. Des séances massives de consultation individuelle des habitants propriétaires de maisons, sous la forme d'entretiens d'une heure réalisés par des architectes, ont permis la " co-conception " et le « co-assemblage " d'une multitude de scénarios partiels de transformation des parcelles privées, au fur et à mesure de l'avancée des réflexions des habitants, éclairées par une modélisation 3D réalisée en temps réel par l'architecte-médiateur. Et c'est l'une des découvertes majeures du projet : par un tel dispositif, six ménages sur dix reçus repartent en ayant fait dessiner à leur architecte un voire deux logements supplémentaires sur leur terrain, soit un gisement qui dépasse largement les besoins en construction de logements de la plupart des communes françaises.

\section{BIBLIOGRAPHIE}

- Christopher Alexander et al., A Pattern Language, New York, Oxford University Press, 1977.

- Jean-Louis Le Moigne, La modélisation des systèmes complexes, Paris, Dunod, 1999.

- Kevin Lynch, Good City Form, Cambridge (Mass.), The Miт Press, 1981.
- David Miet, Benoît Le Foll, « Les modèles de conception : éléments basiques d'une connaissance architecturale et urbanistique partagée ", actes de la conférence Urban Engeneering, Lille, 2005.
- Edgar Morin, La méthode, vol. 1 à 5, Paris, Le Seuil, 1981-2003.

\author{
- Herbert Simon, Les sciences de \\ I'artificiel, Paris, Gallimard, 2004
}

